\title{
Repurposing Avermectins and Milbemycins against Mycobacteroides abscessus and Other Nontuberculous Mycobacteria
}

\author{
Lara Muñoz-Muñoz ${ }^{1,2, *}$, Carolyn Shoen ${ }^{3}$, Gaye Sweet ${ }^{4}$, Asunción Vitoria ${ }^{1,2}$, Tim J. Bull ${ }^{5}$, Michael Cynamon ${ }^{3}$, \\ Charles J. Thompson ${ }^{4}$ and Santiago Ramón-García ${ }^{1,6,7, *(1)}$ \\ 1 Department of Microbiology, Faculty of Medicine, University of Zaragoza, 50009 Zaragoza, Spain; \\ avitagreda@gmail.com \\ 2 Microbiology Unit, Clinical University Hospital Lozano Blesa, 50009 Zaragoza, Spain \\ 3 State University of New York Upstate Medical Center, Syracuse, NY 13210, USA; shoenc@cnyrc.org (C.S.); \\ Michael.Cynamon@va.gov (M.C.) \\ 4 Department of Microbiology and Immunology, Centre for Tuberculosis Research, Life Sciences Institute, \\ University of British Columbia, Vancouver, BC V6T 1Z3, Canada; gsweet@mail.ubc.ca (G.S.); \\ cthompso@mail.ubc.ca (C.J.T.) \\ 5 Institute for Infection \& Immunity, St. George's University of London, London SW17 ORE, UK; \\ tbull@sgul.ac.uk \\ 6 Research \& Development Agency of Aragón (ARAID) Foundation, 50018 Zaragoza, Spain \\ 7 CIBER Enfermedades Respiratorias (CIBERES), Instituto de Salud Carlos III, 28029 Madrid, Spain \\ check for
} updates

Citation: Muñoz-Muñoz, L.; Shoen, C.; Sweet, G.; Vitoria, A.; Bull, T.J.; Cynamon, M.; Thompson, C.J.; Ramón-García, S. Repurposing Avermectins and Milbemycins against Mycobacteroides abscessus and Other Nontuberculous Mycobacteria. Antibiotics 2021, 10, 381. https:// doi.org/10.3390/antibiotics10040381

Academic Editors: Jorge H. Leitão and Luis G. Alves

Received: 19 March 2021

Accepted: 31 March 2021

Published: 3 April 2021

Publisher's Note: MDPI stays neutral with regard to jurisdictional claims in published maps and institutional affiliations.

Copyright: (C) 2021 by the authors Licensee MDPI, Basel, Switzerland. This article is an open access article distributed under the terms and conditions of the Creative Commons Attribution (CC BY) license (https:// creativecommons.org/licenses/by/ $4.0 /)$.

\begin{abstract}
Infections caused by nontuberculous mycobacteria (NTM) are increasing worldwide, resulting in a new global health concern. NTM treatment is complex and requires combinations of several drugs for lengthy periods. In spite of this, NTM disease is often associated with poor treatment outcomes. The anti-parasitic family of macrocyclic lactones (ML) (divided in two subfamilies: avermectins and milbemycins) was previously described as having activity against mycobacteria, including Mycobacterium tuberculosis, Mycobacterium ulcerans, and Mycobacterium marinum, among others. Here, we aimed to characterize the in vitro anti-mycobacterial activity of ML against a wide range of NTM species, including Mycobacteroides abscessus. For this, Minimum Inhibitory Concentration (MIC) values of eight ML were determined against 80 strains belonging to nine different NTM species. Macrocyclic lactones showed variable ranges of anti-mycobacterial activity that were compound and species-dependent. Milbemycin oxime was the most active compound, displaying broad-spectrum activity with MIC lower than $8 \mathrm{mg} / \mathrm{L}$. Time kill assays confirmed MIC data and showed bactericidal and sterilizing activity of some compounds. Macrocyclic lactones are available in many formulations and have been extensively used in veterinary and human medicine with suitable pharmacokinetics and safety properties. This information could be exploited to explore repurposing of anti-helminthics for NTM therapy.
\end{abstract}

Keywords: avermectins; nontuberculous mycobacteria; Mycobacteroides abscessus; selamectin; milbemycin oxime; repurposing

\section{Introduction}

The incidence of documented infections caused by nontuberculous mycobacteria (NTM) are on the rise worldwide and becoming a new neglected global health concern [1-3]. Reasons for this include NTM outbreaks in cosmetic and surgical procedures, potential transmission of Mycobacteroides abscessus among patients with cystic fibrosis (CF), and the increasing number of vulnerable individuals at risk of developing these infections, i.e., immunocompromised patients or patients with chronic pulmonary pathologies [4-6]. The true global burden of NTM disease is vastly under-diagnosed due to several unresolved 
obstacles, including the non-specific presentation of NTM disease and limited diagnostic capacity in many countries, leading to under diagnosis [1-3].

NTM are an extremely diverse group, with more than 190 species currently identified and new species being frequently reported $[7,8]$. Most common diseases caused by NTM are pulmonary, disseminated, or skin and soft tissues infections $[9,10]$. Pulmonary NTM infections are typically caused by Mycobacterium avium-intracellulare complex (MAC), Mycobacterium kansasii, and M. abscessus complex (MABSC) [11,12]. Disseminated infections can be found in immunocompromised patients, including those that have received an organ transplant or have HIV-infection. Skin and soft tissue infections are often associated with trauma, surgical procedures, or contaminated medical equipment with MABSC, Mycobacterium chelonae, Mycobacterium fortuitum, Mycobacterium marinum, and Mycobacterium ulcerans as the most prevalent pathogenic NTM [4,11].

NTM are intrinsically resistant to most antibiotics used in the clinic and patients infected with NTM require combination treatments with a minimum of 2-3 antibiotics for several months (at least 12 months therapy for respiratory or disseminated) and 4-6 months for skin infections $[2,8-10]$. Such prolonged regimens are difficult to tolerate and compliance is challenging $[3,8,13]$. In spite of these aggressive and lengthy treatments, cure rates are low: only 50 to $88 \%$ of MAC patients and 25 to $58 \%$ of MABSC patients achieve sputum culture conversion in respiratory disease [2]. There is an urgent need to identify new compounds that could be used against infections caused by NTM to make therapy more effective and reduce its duration [13].

Macrocyclic lactones (ML) are a family of known anti-parasitics divided into two subfamilies: avermectins and milbemycins. Avermectins are produced by Streptomyces avermitilis and include: ivermectin, abamectin, emamectin, selamectin, doramectin, and eprinomectin. Milbemycins, derived from metabolites produced by Streptomyces hygroscopicus and Streptomyces cyanogriseus, include milbemycin oxime and moxidectin [14,15]. Both families have a common pharmacophore, a 16-member macrocyclic lactone ring [15]. To exert their anti-parasitic function, ML bind to glutamate-gated chloride channels, causing paralysis in nematodes, insects and arachnids [16]. Due to its broad spectrum and its unique pharmacological and safety profile, ML are widely used in veterinary medicine against endo and ectoparasites in pets and livestock [17]. In humans, ivermectin has been widely used over the last 30 years in mass medication campaigns for the treatment and elimination of human onchocerciasis and lymphatic filariasis [18-20] alongside other human diseases [16,19]. Recently, moxidectin was also approved for the treatment of onchocerciasis by the US Food and Drug Administration (FDA) [21]. Researchers continue to explore new applications for ivermectin to reduce malaria, leishmaniasis, and trypanosomiasis transmission by vector control [20,22], or as anti-viral agents against an extensive range of RNA viruses such as HIV-1, Dengue virus, or SARS-CoV-2, among others [23,24]. However, caution is needed when efficacy assessment is based solely on in vitro data [25].

Initially thought to be inactive against bacteria, we previously identified the bactericidal activity of ML against certain mycobacterial species, including Mycobacterium tuberculosis, Mycobacterium bovis, Mycobacterium smegmatis, M. ulcerans and M. marinum, [26,27]. The aim of this study was to expand previous reports to include a wider range of mycobacterial species and evaluate the in vitro anti-mycobacterial activity of ML against NTM.

\section{Results}

2.1. Milbemycin Oxime Was the Most Active Macrocyclic Lactone against NTM with Broad-Spectrum Antimycobacterial Activity

Eight ML were tested against a panel of 80 NTM, including reference strains and clinical isolates from local hospitals (Figure 1, Table S1). Milbemycin oxime was the most active ML against all the species tested: M. abscessus, M. chelonae, and M. fortuitum showed the highest Minimum Inhibitory Concentration (MIC) in a mean range of 4-16 mg/L; M. avium, 2-16 mg/L and the remaining NTM species with MIC values lower than $4 \mathrm{mg} / \mathrm{L}$. Emamectin and selamectin also showed promising activity, although they were less potent or had a narrower activity spectrum than milbemycin oxime. In the case of selamectin, 
MIC distribution was variable with good activity against most species (MIC $\leq 8 \mathrm{mg} / \mathrm{L}$ ) but with no in vitro activity against $M$. abscessus and M. chelonae (MIC $\geq 32 \mathrm{mg} / \mathrm{L}$ ). $M$. avium strains displayed a bimodal MIC distribution ( $\mathrm{MIC}=1-8 \mathrm{mg} / \mathrm{L}$ and $\geq 32 \mathrm{mg} / \mathrm{L}$ ). Ivermectin, abamectin, doramectin, and moxidectin were only moderately active against some strains, with an overall lack of activity (MIC $>32 \mathrm{mg} / \mathrm{L}$ ). Eprinomectin showed no in vitro activity (MIC $>32 \mathrm{mg} / \mathrm{L}$ ).

A.

\begin{tabular}{|c|c|c|c|c|c|c|c|c|c|}
\hline & Strains & ABA & DOR & EMA & EPR & IVM & MBO & Mox & SEL \\
\hline M. abscessus & $n=10$ & & & & & & & & \\
\hline M. avium & $\mathrm{n}=15$ & & & & & & & & \\
\hline M. chelonae & $n=6$ & & & & & & & & \\
\hline M. fortuitum & $n=9$ & & & & & & & & \\
\hline M. gordonae & $n=8$ & & & & & & & & \\
\hline M. intracellulare & $n=6$ & & & & & & & & \\
\hline M. kansasil & $\mathrm{n}=18$ & & & & & & & & \\
\hline M. marinum & $n=5$ & & & & & & & & \\
\hline
\end{tabular}

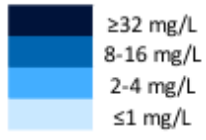

B.
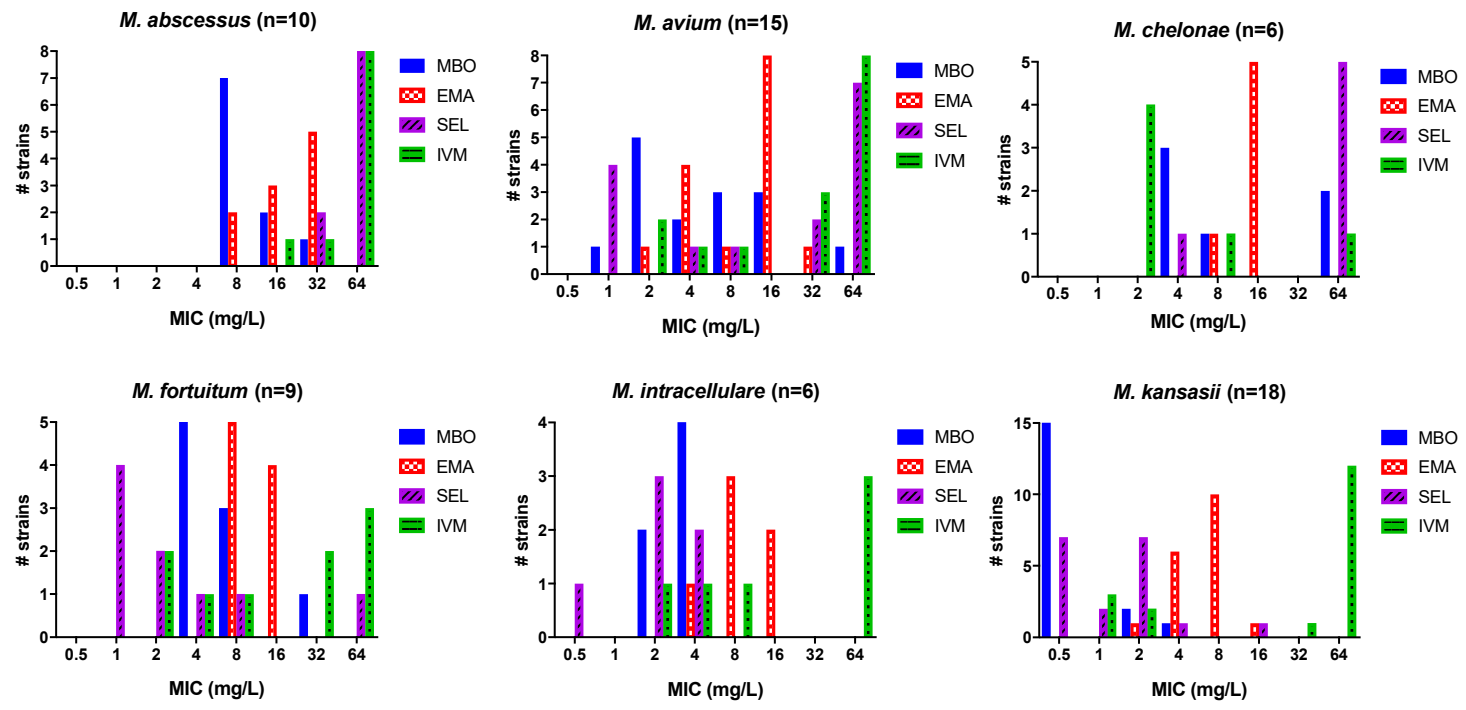

Figure 1. In vitro susceptibility of nontuberculosis mycobacteria (NTM) against different avermectins and milbemycins. (A) Heat map representation of the most common MIC values of eight ML against a panel of NTM strains. (B) MIC distribution of the most active ML against clinically relevant NTM strains. ABA, abamectin; DOR, doramectin; EMA, emamectin; EPR, eprinomectin; IVM, ivermectin; MBO, milbemycin oxime; MOX, moxidectin; SEL, selamectin.

\subsection{Milbemycin Oxime Did Not Show any Inducible Resistance against Rapidly Growing Mycobacteria, in Contrast to Clarithromycin}

ML are structurally similar to antibacterial macrolides - both contain a macrocyclic lactone ring $[15,18,28]$. Current CLSI guidelines recommend performing a second MIC measurement after an extended incubation period of at least 14 days in order to detect inducible macrolide resistance in rapidly growing mycobacteria (RGM) [29]. We performed MIC determinations after the standard 3 days and extended 14 days of incubation to determine whether milbemycin oxime displayed a similar inducible resistance pattern as the first-line macrolide antibiotic clarithromycin (Table 1). Consistent with the distribution of the macrolide inducible resistance erm genes in the different species, M. abscessus sp. abscessus, M. abscessus sp. bolletii, and M. fortuitum showed a strong increase in their clarithromycin MIC values after 14 days of incubation compared to values after 3 days; this was not observed for M. abscessus sp. massiliense, or M. chelonae [30-33]. In contrast, MIC values of milbemycin oxime were the same against different species at both incubation times. 


\subsection{Macrocyclic Lactones Displayed Selective Dose-Dependent Bacteriostatic or Bactericidal Activities against Different NTM Species}

To further characterize the in vitro activity of ML against NTM, we performed Time Kill Assays (TKA) of milbemycin oxime (Figure 2), ivermectin and selamectin (Figure S1) at two concentrations against some clinically relevant NTM species; the first-line drug clarithromycin was included as an internal control. TKA of clarithromycin explained the observed increase in MIC values at 3 and 14 days (Table 1), i.e., there was a growth rebound in M. abscessus sp. abscessus, M. abscessus sp bolletii and, especially, M. fortuitumprobably due to the development of inducible resistance against clarithromycin. In the case of M. abscessus sp. massiliense and M. chelonae, lacking a macrolide inducible erm resistance gene, both concentrations of clarithromycin showed rapid bactericidal and sterilizing activity [29-33]. For those species with macrolide inducible resistance, milbemycin oxime $80 \mathrm{mg} / \mathrm{L}$ exhibited the best activity, preventing regrowth in M. abscessus sp. bolletii and $M$. fortuitum. Importantly, milbemycin oxime was equally or slightly more active than clarithromycin against $M$. abscessus sp. abscessus. Milbemycin oxime was especially bactericidal against $M$. kansasii with sterilizing activity at both concentrations tested, but bacteriostatic against M. avium and M. intracellulare (although at $80 \mathrm{mg} / \mathrm{L}$ the effect was prolonged, suggesting that re-dosing could increase activity) (Figure 2). The anti-mycobacterial activities of selamectin and ivermectin were also characterized by TKA against RGM, displaying a reduced activity compared to milbemycin oxime. While ivermectin was inactive, selamectin showed some activity against $M$. chelonae and $M$. fortuitum but not against MABSC. Both concentrations of selamectin displayed a bacteriostatic profile against slow-growing mycobacteria (SGM) (Figure S1).

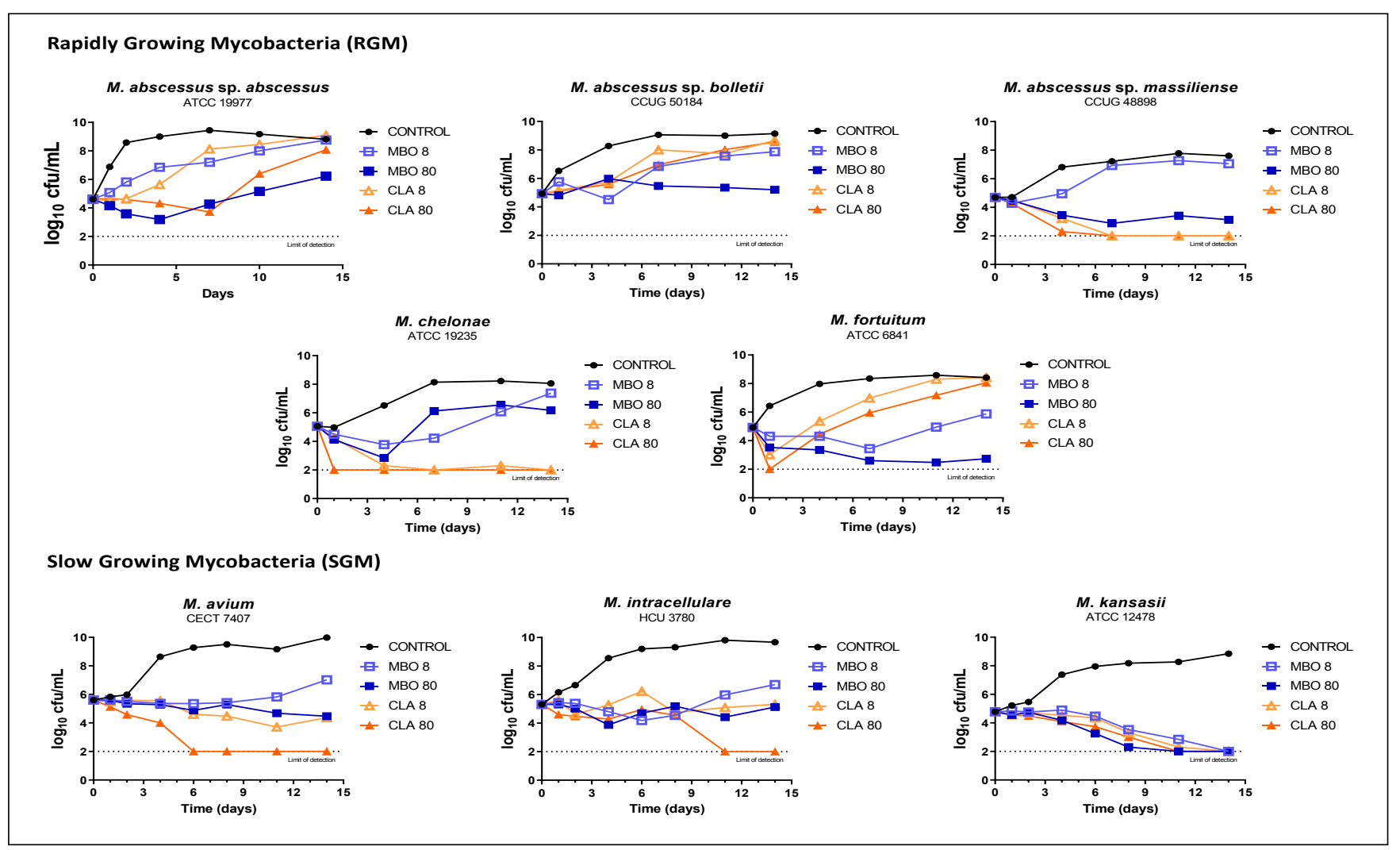

Figure 2. In vitro Time Kill Assays of milbemycin oxime against different RGM and SGM species. Clarithromycin was also included as internal control of activity. Compounds were tested at $8 \mathrm{mg} / \mathrm{L}$ and $80 \mathrm{mg} / \mathrm{L}$, i.e., $1 \times \mathrm{MIC}$ and $10 \times \mathrm{MIC}$ of milbemycin oxime, respectively. MBO, milbemycin oxime; CLA, clarithromycin. 
Table 1. Differential MIC values of selected compounds against rapidly growing mycobacteria (RGM) after 3 and 14 days of incubation. Amikacin was included as a control antibiotic not containing a macrocyclic lactone ring. MBO, milbemycin oxime; CLA, clarithromycin; AMK, amikacin.

\begin{tabular}{|c|c|c|c|c|c|c|c|c|c|}
\hline \multirow{3}{*}{ Strains } & \multicolumn{9}{|c|}{ MIC (mg/L) for RGM Strains } \\
\hline & \multicolumn{2}{|c|}{ MBO } & \multirow{2}{*}{$\begin{array}{c}\text { Fold } \\
\text { Change }\end{array}$} & \multicolumn{2}{|c|}{ CLA } & \multirow{2}{*}{$\begin{array}{c}\text { Fold } \\
\text { Change }\end{array}$} & \multicolumn{2}{|c|}{ AMK } & \multirow{2}{*}{$\begin{array}{c}\text { Fold } \\
\text { Change }\end{array}$} \\
\hline & 3 Days & 14 Days & & 3 Days & 14 Days & & 3 Days & 14 Days & \\
\hline M. abscessus sp. abscessus ATCC 19977 & 8 & 8 & 1 & 4 & 128 & 32 & 16 & 64 & 4 \\
\hline M. abscessus sp. bolletii CCUG 50184 & 8 & 8 & 1 & 4 & 128 & 32 & 16 & 32 & 2 \\
\hline M. abscessus sp. masiliense CCUG 48898 & 8 & 8 & 1 & 0.5 & 2 & 4 & 16 & 64 & 4 \\
\hline M. chelonae ATCC 19235 & 8 & 16 & 2 & 0.12 & 0.5 & 4 & nd & nd & nd \\
\hline M. fortuitum ATCC 6841 & 8 & 8 & 1 & 8 & 64 & 8 & 4 & 4 & 1 \\
\hline
\end{tabular}

\section{Discussion}

Macrolide antibiotics are the backbone of current NTM therapies, typically administered in combination with amikacin [7,34]. However, the development of macrolide resistance occurs frequently, thus restricting their therapeutic efficacy. Resistance to macrolides monitored in vitro is associated with poorer outcomes $[10,13]$. Although there have been some recent advances in NTM therapy, there has not been much improvement regarding new drugs or regimens, since the macrolide-based multidrug therapy was established in 1990 [2]; this could be, in part, due to the traditionally reduced interest from pharmaceutical companies in developing specific NTM therapies, together with the lack of both awareness of this neglected problem and robust data defining the global burden of NTM disease [1]. There is thus an urgent need to identify new drugs and develop more effective and safer NTM regimens, especially to treat infections resistant to macrolides. We previously demonstrated the in vitro activity of some ML against mycobacteria, including pathogenic M. tuberculosis and M. ulcerans [26,27]. Here, we characterized the potential role of ML against NTM infections.

The in vitro antimicrobial activities of eight ML were tested against a panel of 80 NTM strains including nine species (Figure 1, Table S1). Milbemycin oxime was the most active (MIC $\leq 8 \mathrm{mg} / \mathrm{L}$ ), with broad-spectrum activity against all species tested. In the case of selamectin (the compound with the best potential based on available pharmacokinetic properties [27]) the MIC distribution was variable, with good activity against most species (MIC $\leq 8 \mathrm{mg} / \mathrm{L}$ ), but with limited in vitro activity against $M$. abscessus and $M$. chelonae $(\mathrm{MIC} \geq 32 \mathrm{mg} / \mathrm{L})$.

A major concern in NTM therapy is the development of macrolide resistance conferred by two well-known genes: $\mathrm{rrl}$ and $\mathrm{erm}$ [35]. Mutations in the $r r l$ gene providing macrolide resistance rarely emerge during therapy. In contrast, macrolide-inducible erm-dependent resistance is common in NTM therapy, with erm genes identified in several RGM [30,32,35]. Subspecies of $M$. abscessus differ at the erm41 locus; in erm41, the T polymorphism at nucleotide position 28 (T28) of the structural gene confers inducible resistance, while isolates with the $\mathrm{C}$ polymorphism (C28) remain susceptible. M. abscessus sp. bolletii includes the T28 polymorphism, M. abscessus sp. abscessus can have either (T28 or C28), and the erm 41 gene in the M. abscessus sp. massiliense is truncated and non-functional [30]. In $M$. chelonae these mutations have not been observed, while $M$. fortuitum can express the inducible erm39 isoform [31,32]. CLSI guidelines recommend reporting MIC values after 3 and 14 days of incubation to identify clarithromycin resistance due to an erm induction mechanism [29]. In our study, we confirmed these observations: M. abscessus sp. abscessus, M. abscessus sp. bolleti, and M. fortuitum showed an MIC increase from day 3 to day 14; in contrast, $M$. abscessus sp. masiliense and M. chelonae did not show this increase (Table 1). ML are structurally similar to macrolide antibiotics, both containing a macrocyclic lactone ring $[15,18,28] ;$ ML are 16-membered macrocyclic lactone ring compounds, while macrolides could have 12-, 14-, 15-, or 16-membered macrocyclic lactone rings [28]. We thus investigated whether resistance to ML could also be inducible in RGM, similar to clarithromycin. We found that the MIC values of milbemycin oxime remained constant at 
both 3 and 14 days of incubation in all five strains tested, suggesting a lack of an inducible resistant mechanism (Table 1). To further understand the antimicrobial activity of ML, we also performed TKA in parallel with the first line drug clarithromycin. ML displayed a bacteriostatic or bactericidal activity dependent on the NTM species tested. Importantly, a detailed analysis of the time-kill kinetic curves revealed better or similar potency of milbemycin oxime and clarithromycin against some of the strains (Figure 2). In the case of M. fortuitum, for which the induction of clarithromycin resistance was more evident (regrowth after only one day of drug exposure), at a high dose $(80 \mathrm{mg} / \mathrm{L})$, milbemycin oxime was able to prevent regrowth after 14 days of incubation.

ML are orally active compounds with fast absorption [14], wide distribution in the body, and long residence times $[18,19]$. Due to their specificity for parasitic targets they have a high margin of safety in mammals at clinical doses [36]. Ivermectin and moxidectin are the only ML approved for human use, ivermectin being the most widely used. At clinical doses, ivermectin is extremely well tolerated and safe [37]. Similarly, moxidectin, recently approved for humans, has been widely used in veterinary medicine. However, in this study, neither of these compounds showed significant activity against any of the mycobacterial strains tested. In contrast, milbemycin oxime, the most active of the ML according to in vitro data, and selamectin, the ML with the best translational potential based on PK considerations [27], displayed significant activity in our study. However, at present, these are commercialized and licensed only for veterinary use (cats and dogs) [17]. Their inclusion in NTM therapy would thus require a repurposing approach that could be facilitated thanks to the extensive pharmacological data available from animal studies.

Milbemycin oxime reaches higher concentrations in blood than ivermectin with an improved safety profile due to reduce P-gp binding [27]. At half $(0.25 \mathrm{mg} / \mathrm{kg})$ and double $(0.92 \mathrm{mg} / \mathrm{kg})$ the standard dose of milbemycin oxime typically used in dogs, $\mathrm{C}_{\max }$ values could achieve $79.33 \mu \mathrm{g} / \mathrm{L}$ and $353 \mu \mathrm{g} / \mathrm{L}$, respectively [27]. These concentrations are ca. 25-fold lower than our observed effective dosing for anti-mycobacterial activity (ca. $8 \mathrm{mg} / \mathrm{L}$ ); thus, simple extrapolation would approximate a potential effective dose to be about $25 \mathrm{mg} / \mathrm{kg}$, although appropriate projections should be calculated to this end. Previous work has shown a dose ca. 100 -fold higher $(100 \mathrm{mg} / \mathrm{kg})$ caused severe, adverse, but transient effects in mice while doses of $300 \mathrm{mg} / \mathrm{kg}$ produced severe toxicosis and death [38]. In the case of selamectin, oral doses of $24 \mathrm{mg} / \mathrm{kg}$ have been tested reaching $\mathrm{C}_{\max }$ values of $7.6 \mathrm{mg} / \mathrm{L}$ [39]; this is within the effective anti-mycobacterial range. In toxicity studies performed in mice, doses of $30 \mathrm{mg} / \mathrm{kg}$ of selamectin did not cause any adverse effect, while only mild toxicity signs were observed at $100 \mathrm{mg} / \mathrm{kg}$ and $300 \mathrm{mg} / \mathrm{kg}$ [38]. Although milbemycin oxime may have some margin for a safe dose increase, without detailed human dose prediction studies, current information suggests that selamectin could be the ML of choice for some NTM infections.

\section{Materials and Methods}

\subsection{Mycobacterial Strains}

Eighty NTM strains (within nine species) were used: $M$. abscessus $(\mathrm{n}=10)$, M. avium $(\mathrm{n}=15)$, M. chelonae $(\mathrm{n}=6)$, M. fortuitum $(\mathrm{n}=9), M$. gordonae $(\mathrm{n}=8), M$. intracellulare $(\mathrm{n}=6)$, M. kansasii $(\mathrm{n}=18), M$. marinum $(\mathrm{n}=5)$, and M. microti $(\mathrm{n}=3)$. Reference strains were procured from strain collections: from American Type Culture Collection M. abscessus sp. abscessus ATCC 19977, M. chelonae ATCC 19235, M. fortuitum ATCC 6841, M. intracellulare ATCC 35761, M. kansasii ATCC 12478; from Spanish Type Culture Collection M. avium CECT 7404; and from Culture Collection University of Gothenburg M. abscessus sp. bolletii CCUG 50184 and M. abscessus sp. masiliense CCUG 48898. Clinical strains were provided by the Clinical University Hospital Lozano Blesa, Zaragoza, Spain; by the Veterans Affairs Medical Center, Syracuse, New York; by St George's University of London, UK; and by the University of British Columbia, Vancouver, Canada. Species were identified using GenoType Mycobacterium CM/AS assay (Hain Lifescience $\mathrm{GmbH}$, Nehren, Germany) and PCR-restriction fragment length polymorphism analysis [40]. 


\subsection{General Growth Conditions and Drugs}

Strains were grown in Middlebrook 7H9 broth (Difco) supplemented with 10\% albumin, dextrose, and catalase (ADC) (Difco), and 0.5\% glycerol. Middlebrook 7H10 agar plates (Difco) supplemented with 10\% oleic acid, albumin, dextrose, and catalase (OADC) (Difco) and $0.2 \%$ glycerol were used for bacterial CFU enumeration. All strains were incubated at $35-37^{\circ} \mathrm{C}$. Compounds (and suppliers) were: abamectin, doramectin, clarithromycin, amikacin, and linezolid (Sigma-Aldrich, Steinheim, Germany); emamectin and eprinomectin (LKT Labs, Minnesota, MN, USA); ivermectin (Alpha Diagnostic, Texas, TX, USA); and milbemycin oxime, moxidectin, and selamectin (European Pharmacopeia). Stocks solutions $(10 \mathrm{mg} / \mathrm{mL}$ ) were dissolved in DMSO (except amikacin, which was dissolved in sterile water), aliquoted, and stored at $-80^{\circ} \mathrm{C}$ until use.

\subsection{Drug Susceptibility Testing}

MIC determinations were performed by broth microdilution assays in a 96-well plate format by serial 2-fold dilutions of test compounds as previously described [26]. Briefly, mycobacteria were added to a final cell density of $5 \times 10^{5}$ cells $/ \mathrm{mL}$. Positive and negative growth controls were included in every plate for each strain. Plates were incubated at $35-37^{\circ} \mathrm{C}$ for two days (and thirteen days in the case of clarithromycin) for the RGM and five days for the SGM. After the incubation period, the redox indicator MTT (3-(4,5-dimethylthiazol-2-yl)-2,5-diphenyl tetrazolium bromide) was added to the wells and incubated overnight. Then, optical density was read at $580 \mathrm{~nm}$ to measure the MTT to formazan conversion, an indicator of bacterial growth. The lowest drug concentration that inhibited conversion by $90 \%$ compared to the internal negative control was used to define the MIC value. Experiments were done in duplicate at least three times.

\subsection{Time Kill Assays}

Bacterial cultures were inoculated at a final cell density of $10^{5}$ cells $/ \mathrm{mL}$ and a final volume of $10 \mathrm{~mL}$ media in $25 \mathrm{~cm}^{2}$ tissue culture flasks. Drugs were added at designated concentrations based on previously calculated MIC values. Flasks were then incubated at $35-37^{\circ} \mathrm{C}$ for 14 days. At every time point, bacterial suspensions were thoroughly mixed, serially diluted 10 -fold in PBS with $0.1 \%$ tyloxapol (Sigma-Aldrich), and $100 \mu \mathrm{L}$ aliquots plated on agar plates. CFUs were enumerated after 3 days of incubation for RGM and 9 days for SGM at $35-37^{\circ} \mathrm{C}$. Plates were checked again for late growers. A growth control (no drug) was always included for each strain. Experiments were performed in duplicate at least twice.

\section{Conclusions}

The surge in NTM infection incidence and poor treatment outcomes highlights an urgent need to identify new therapeutic alternatives. We profiled in vitro activities of several ML against a panel of NTM pathogens, including M. avium, M. kansasii, and M. abscessus that typically caused pulmonary NTM infections. Milbemycin oxime displayed the best antimycobacterial profile. Selamectin also had good activity and could offer a more valid alternative based on its PK properties. Although these compounds are currently only licensed for veterinary use, the extensive pharmacological data available in different animal species could facilitate a repurposing drug development approach.

Supplementary Materials: The following are available online at https://www.mdpi.com/article/10 .3390 / antibiotics10040381/s1, Table S1. Antibacterial activity of ML against different NTM species. Figure S1. In vitro Time Kill Assays of selamectin and ivermectin against different RGM and SGM species. 
Author Contributions: Conceptualization, C.J.T. and S.R.-G.; Data curation, L.M.-M. and S.R.-G.; Formal analysis, L.M.-M. and S.R.-G.; Funding acquisition, C.J.T. and S.R.-G.; Methodology, L.M.-M., C.S. and G.S.; Resources, A.V., T.J.B., M.C. and C.J.T.; Supervision, T.J.B., M.C., C.J.T. and S.R.-G.; Writing-original draft, L.M.-M. and S.R.-G.; Writing—review and editing, L.M.-M., G.S., T.J.B., M.C., C.J.T. and S.R.-G. All authors have read and agreed to the published version of the manuscript.

Funding: This research was funded by a fellowship from the Government of Aragon (Gobierno de Aragón y Fondos FEDER de la Unión Europea “Construyendo Europa desde Aragón”) to L.M.-M., by a grant from Canadian Institutes of Health Research (MOP-82855) grant to C.J.T. and, by grant from the European Society of Clinical Microbiology and Infectious Diseases (ESCMID Research Grants) and a grant from the Government of Aragon, Spain (Ref. LMP132_18) (Gobierno de Aragón y Fondos Feder de la Unión Europea “Construyendo Europa desde Aragón”) to S.R.-G.

Institutional Review Board Statement: Not applicable.

Informed Consent Statement: Not applicable.

Data Availability Statement: The data presented in this study are available in the article or supplementary material.

Acknowledgments: The results described in this manuscript were partially presented as a poster communication at the 29th European Congress of Clinical Microbiology and Infectious Diseases (Abstract ID 5265).

Conflicts of Interest: The authors declare no conflict of interest.

\section{References}

1. Ahmed, I.; Tiberi, S.; Farooqi, J.; Jabeen, K.; Yeboah-Manu, D.; Migliori, G.B.; Hasan, R. Non-Tuberculous Mycobacterial Infections-A Neglected and Emerging Problem. Int. J. Infect. Dis. 2020, 92, S46-S50. [CrossRef] [PubMed]

2. Wu, M.L.; Aziz, D.B.; Dartois, V.; Dick, T. NTM Drug Discovery: Status, Gaps and the Way Forward. Drug Discov. Today 2018, 23, 1502-1519. [CrossRef] [PubMed]

3. Ratnatunga, C.N.; Lutzky, V.P.; Kupz, A.; Doolan, D.L.; Reid, D.W.; Field, M.; Bell, S.C.; Thomson, R.M.; Miles, J.J. The Rise of Non-Tuberculosis Mycobacterial Lung Disease. Front. Immunol. 2020, 11, 1-12. [CrossRef]

4. Muñoz-Egea, M.C.; Carrasco-Antón, N.; Esteban, J. State-of-the-Art Treatment Strategies for Nontuberculous Mycobacteria Infections. Expert Opin. Pharmacother. 2020, 6566. [CrossRef] [PubMed]

5. Guglielmetti, L.; Mougari, F.; Lopes, A.; Raskine, L.; Cambau, E. Human Infections Due to Nontuberculous Mycobacteria: The Infectious Diseases and Clinical Microbiology Specialists ' Point of View. Future Microbiol. 2015, 10, 1467-1483. [CrossRef]

6. Degiacomi, G.; Sammartino, J.C.; Chiarelli, L.R.; Riabova, O.; Makarov, V.; Pasca, M.R. Mycobacterium Abscessus, an Emerging and Worrisome Pathogen among Cystic Fibrosis Patients. Int. J. Mol. Sci. 2019, 20, 5868. [CrossRef]

7. Bento, C.M.; Gomes, M.S.; Silva, T. Looking beyond Typical Treatments for Atypical Mycobacteria. Antibiotics 2020, 9, 18. [CrossRef]

8. Daley, C.L.; Iaccarino, J.M.; Lange, C.; Cambau, E.; Wallace, R.J.; Andrejak, C.; Böttger, E.C.; Brozek, J.; Griffith, D.E.; Guglielmetti, L.; et al. Treatment of Nontuberculous Mycobacterial Pulmonary Disease: An Official ATS/ERS/ESCMID/IDSA Clinical Practice Guideline. Eur. Respir. J. 2020, 56. [CrossRef]

9. Da Mata-Jardín, O.; Angulo, A.; Rodríguez, M.; Fernández-Figueiras, S.; de Waard, J.H. Drug Susceptibility Patterns of Rapidly Growing Mycobacteria Isolated from Skin and Soft Tissue Infections in Venezuela. Eur. J. Clin. Microbiol. Infect. Dis. 2020, 39, 433-441. [CrossRef]

10. Esteban, J.; Navas, E. Tratamiento de Las Infecciones Producidas Por Micobacterias No Tuberculosas. Enferm. Infecc. Microbiol. Clin. 2018, 36, 586-592. [CrossRef]

11. Winburn, B.; Sharman, T. Atypical Mycobacterial Disease Pathophysiology. Clin. Med. 2020, 13, 1-8.

12. Johansen, M.D.; Herrmann, J.L.; Kremer, L. Non-Tuberculous Mycobacteria and the Rise of Mycobacterium Abscessus. Nat. Rev. Microbiol. 2020. [CrossRef] [PubMed]

13. Adelman, M.H.; Addrizzo-Harris, D.J. Management of Nontuberculous Mycobacterial Pulmonary Disease. Curr. Opin. Pulm. Med. 2018, 24, 212-219. [CrossRef] [PubMed]

14. Merola, V.M.; Eubig, P.A. Toxicology of Avermectins and Milbemycins (Macrocyclic Lactones) and the Role of P-Glycoprotein in Dogs and Cats. Vet. Clin. N. Am. Small Anim. Pract. 2018, 48, 991-1012. [CrossRef] [PubMed]

15. Prichard, R.; Ménez, C.; Lespine, A. Moxidectin and the Avermectins: Consanguinity but Not Identity. Int. J. Parasitol. Drugs Drug Resist. 2012, 2, 134-153. [CrossRef] [PubMed]

16. Długońska, H. The Nobel Prize 2015 in Physiology or Medicine for Highly Effective Antiparasitic Drugs. Ann. Parasitol. 2015, 61, 299-301.

17. Nolan, J.; Lok, T.B.J. Macrocyclic Lactones in the Treatment and Control of Parasitism in Small Companion Animals. Curr. Pharm. Biotechnol. 2012, 13, 1078-1094. [CrossRef]

18. McKellar, Q.A.; Benchaoui, H.A. Avermectins and Milbemycins. J. Vet. Pharmacol. Ther. 1996, 19, 331-351. [CrossRef] 
19. Canga, A.G.; Prieto, A.M.S.; Diez Liébana, M.J.; Martínez, N.F.; Sierra Vega, M.; García Vieitez, J.J. The Pharmacokinetics and Interactions of Ivermectin in Humans-A Mini-Review. AAPS J. 2008, 10, 42-46. [CrossRef]

20. Crump, A. Ivermectin: Panacea for Resource-Poor Communities? Trends Parasitol. 2014, 30, 445-455.

21. FDA U.S. Food \& Drug Administration. Available online: Https://Www.Accessdata.Fda.Gov/Drugsatfda_docs/Label/2018/2 10867lbl.Pdf (accessed on 2 April 2021).

22. Roadmappers, T.I. A Roadmap for the Development of Ivermectin as a Complementary Malaria Vector Control Tool. Am. J. Trop. Med. Hyg. 2020, 102, 3-24. [CrossRef] [PubMed]

23. Wagstaff, K.M.; Sivakumaran, H.; Heaton, S.M.; Harrich, D.; Jans, D.A. Ivermectin Is a Specific Inhibitor of Importin $\alpha / \beta$-Mediated Nuclear Import Able to Inhibit Replication of HIV-1 and Dengue Virus. Biochem. J. 2012, 443, 851-856. [CrossRef] [PubMed]

24. Caly, L.; Druce, J.D.; Catton, M.G.; Jans, D.A.; Wagstaff, K.M. The FDA-Approved Drug Ivermectin Inhibits the Replication of SARS-CoV-2 in Vitro. Antiviral Res. 2020, 104787. [CrossRef] [PubMed]

25. Chaccour, C.; Hammann, F.; Ram, S. Editorial Ivermectin and Novel Coronavirus Disease ( COVID-19 ): Keeping Rigor in Times of Urgency. Am. J. Trop. Med. Hyg. 2020,1-2. [CrossRef]

26. Lim, L.E.; Vilchèze, C.; Ng, C.; Jacobs, W.R.; Ramón-García, S.; Thompson, C.J. Anthelmintic Avermectins Kill Mycobacterium Tuberculosis, Including Multidrug-Resistant Clinical Strains. Antimicrob. Agents Chemother. 2013, 57, 1040-1046. [CrossRef] [PubMed]

27. Scherr, N.; Pluschke, G.; Thompson, C.J.; Ramón-García, S. Selamectin Is the Avermectin with the Best Potential for Buruli Ulcer Treatment. PLoS Negl. Trop. Dis. 2015, 9, 1-10. [CrossRef] [PubMed]

28. Dinos, G.P. The Macrolide Antibiotic Renaissance. Br. J. Pharmacol. 2017, 174, 2967-2983. [CrossRef]

29. Clinical \& Laboratory Standards Institute. Susceptibility Testing of Mycobacteria, Nocardiae, and Other Aerobic Actinomycetes; Approved Standard Second Edition-M24A2; Clinical \& Laboratory Standards Institute: Annapolis Junction, MD, USA, 2011.

30. Nessar, R.; Cambau, E.; Reyrat, J.M.; Murray, A.; Gicquel, B. Mycobacterium Abscessus: A New Antibiotic Nightmare. J. Antimicrob. Chemother. 2012, 67, 810-818. [CrossRef]

31. Hanson, K.E.; Slechta, E.S.; Muir, H.; Barker, A.P. Rapid Molecular Detection of Inducible Macrolide Resistance in Mycobacterium Chelonae and M. Abscessus Strains: A Replacement for 14-Day Susceptibility Testing? J. Clin. Microbiol. 2014, 52, 1705-1707. [CrossRef]

32. Aono, A.; Morimoto, K.; Chikamatsu, K.; Yamada, H. Antimicrobial Susceptibility Testing of Mycobacteroides (Mycobacterium) Abscessus Complex, Mycolicibacterium (Mycobacterium) Fortuitum, and Mycobacteroides (Mycobacterium) Chelonae *. J. Infect. Chemother. 2018, 4-10. [CrossRef]

33. Hatakeyama, S.; Ohama, Y.; Okazaki, M.; Nukui, Y.; Moriya, K. Antimicrobial Susceptibility Testing of Rapidly Growing Mycobacteria Isolated in Japan. BMC Infect. Dis. 2017, 17, 1-7. [CrossRef]

34. Esteban, J.; Martín-de-Hijas, N.Z.; García-Almeida, D.; Bodas-Sánchez, A.; Gadea, I.F.-R.R. Prevalence of Erm Methylase Genes in Clinical Isolates of Non-Pigmented, Rapidly Growing Mycobacteria. Clin. Microbiol. Infect. 2009, 15, 919-923. [CrossRef]

35. Li, F.; Li, G.L.; Pang, H.; Liu, H.C.; Xiao, T.Y.; Li, S.J.; Luo, Q.; Jiang, Y.; Wang, R.B.; Wan, K.L. Preliminary Study on Drug Susceptibility Profile and Resistance Mechanisms to Macrolides of Clinical Isolates of Non-Tuberculous Mycobacteria from China. Biomed. Environ. Sci. 2018, 31, 290-299.

36. Chhaiya, S.; Mehta, D.; Kataria, B. Ivermectin: Pharmacology and Therapeutic Applications. Int. J. Basic Clin. Pharmacol. 2012, 1, 132. [CrossRef]

37. Navarro, M.; Camprubí, D.; Requena-Méndez, A.; Buonfrate, D.; Giorli, G.; Kamgno, J.; Gardon, J.; Boussinesq, M.; Muñoz, J.; Krolewiecki, A. Safety of High-Dose Ivermectin: A Systematic Review and Meta-Analysis. J. Antimicrob. Chemother. 2020, 75, 827-834. [CrossRef]

38. Bishop, B.F.; Bruce, C.I.; Evans, N.A.; Goudie, A.C.; Gration, K.A.F.; Gibson, S.P.; Pacey, M.S.; Perry, D.A.; Walshe, N.D.A.; Witty, M.J. Selamectin: A Novel Broad-Spectrum Endectocide for Dogs and Cats. Vet. Parasitol. 2000, 91, 163-176. [CrossRef]

39. Sarasola, P.; Jernigan, A.D.; Walker, D.K.; Castledine, J.; Smith, D.G.; Rowan, T.G.; McTier, T.L. Pharmacokinetics of Selamectin Following Intravenous, Oral and Topical Administration in Cats and Dogs. J. Vet. Pharmacol. Ther. 2002, 25, 265-272. [CrossRef] [PubMed]

40. Amilio, T.; Francine, M.; Marianne, B.; Frank, B.E.C.B. Rapid Identification of Mycobacteria to the Species Level by Polymerase Chain Reaction and Restriction Enzyme Analysis. J. Clin. Microbiol. 1993, 31, 175-178. 